\title{
Spatial evolution of the kurtosis of steep unidirectional random waves
}

Tang, Tianning; Xu, Wentao; Barratt, Dylan; Bingham, Harry B.; Li, Y.; Taylor, P. H.; van den Bremer, T. S.; Adcock, T. A. A.

Published in:

Journal of Fluid Mechanics

Link to article, DOI:

10.1017/jfm.2020.841

Publication date:

2020

Document Version

Early version, also known as pre-print

Link back to DTU Orbit

Citation (APA):

Tang, T., Xu, W., Barratt, D., Bingham, H. B., Li, Y., Taylor, P. H., van den Bremer, T. S., \& Adcock, T. A. A. (2020). Spatial evolution of the kurtosis of steep unidirectional random waves. Journal of Fluid Mechanics, 908 , [A3]. https://doi.org/10.1017/jfm.2020.841

\section{General rights}

Copyright and moral rights for the publications made accessible in the public portal are retained by the authors and/or other copyright owners and it is a condition of accessing publications that users recognise and abide by the legal requirements associated with these rights.

- Users may download and print one copy of any publication from the public portal for the purpose of private study or research.

- You may not further distribute the material or use it for any profit-making activity or commercial gain

- You may freely distribute the URL identifying the publication in the public portal 


\title{
Spatial evolution of the kurtosis of steep unidirectional random waves
}

\author{
Tianning Tang ${ }^{1}$, Wentao $\mathrm{Xu}^{2}$, Dylan Barratt ${ }^{1}, \mathrm{H}$. B. Bingham ${ }^{3}$, \\ Y. $\mathrm{Li}^{2} \dagger$, P. H. Taylor ${ }^{4,1}$, T. S. van den Bremer ${ }^{1}$ and T. A. A. Adcock ${ }^{1}$ \\ ${ }^{1}$ Department of Engineering Science, University of Oxford, Oxford, OX1 3PJ, UK \\ ${ }^{2}$ School of Naval Architecture, Ocean and Civil Engineering, Shanghai Jiao Tong University, \\ Shanghai, 200240, China \\ ${ }^{3}$ Department of Mechanical Engineering, Technical University of Denmark, Lyngby, DK-2800, \\ Denmark \\ ${ }^{4}$ Faculty of Engineering and Mathematical Sciences, University of Western Australia, Crawley, \\ WA 6009, Australia
}

(Received $\mathrm{xx}$; revised $\mathrm{xx}$; accepted $\mathrm{xx}$ )

We study the evolution of unidirectional water waves from a randomly forced input condition with uncorrelated Fourier components. We examine the kurtosis of the linearised free surface as a convenient proxy for the probability of a rogue wave. We repeat the laboratory experiments of Onorato et al. (2004, Phys. Rev. E 70 (6), 067302), both experimentally and numerically, and extend the parameter space in our numerical simulations. We consider numerical simulations based on the Modified Nonlinear Schrödinger equation (MNLS) and the fully nonlinear water wave equations, which are in good agreement. For low steepness, existing analytical models based on the Nonlinear Schrödinger equation (NLS) are found to be accurate. For cases which are steep or have very narrow bandwidths, these analytical models over-predict the rate at which excess kurtosis develops. In these steep cases, the kurtosis in both our experiments and numerical simulations peaks before returning to an equilibrium level. Such transient maxima are not predicted by NLS-based analytical models. Above a certain threshold of steepness, the steady-state value of kurtosis is primarily dependent on the spectral bandwidth. We also examine how the average shape of extreme events is modified by nonlinearity over the evolution distance, showing significant asymmetry during the initial evolution, which is greatly reduced once the spectrum has reached equilibrium. The locations of the maxima in asymmetry coincide approximately with the locations of the maxima in kurtosis.

\section{Introduction}

The simplest model of free surface gravity waves assumes linear dynamics. In this model, and if the free surface is modelled as a Gaussian random process, wave amplitudes are well approximated by a Rayleigh distribution (Longuet-Higgins 1952), and the average shape of large waves is given by the scaled autocorrelation function (Lindgren 1970; Boccotti 1983). Nonlinearity leads to modifications in the wave statistics and to the shape of extreme wave events. In particular, when the fluid is deep and the waves are unidirectional, the Benjamin-Feir instability (Benjamin \& Feir 1967) leads to more large waves than would be expected from the linear model as correlations develop between Fourier components. A convenient parameter to describe the increased number of large waves is the kurtosis (or excess kurtosis) of the free surface (Mori \& Janssen 2006). Here,

$\dagger$ Email address for correspondence: ye.li@sjtu.edu.cn 
we examine how the kurtosis and average shape of an extreme wave evolve in space from a Gaussian random input condition without correlation between components. The overarching objective is to improve our understanding of the nature of the non-linear physics of surface gravity waves and its impact on wave statistics.

Analysis of this problem originates from Janssen (2003). In this paper the wave field is assumed to be sufficiently weakly nonlinear that the sea surface is in a near-to-Gaussian state, meaning that the kurtosis can be expressed in terms of lower-order moments, following the approach of Hasselmann (1962). Ensemble averaging of the Zakharov equation and the assumption of spatial homogeneity provides closed-form expressions for the fourth cumulant and the evolution of kurtosis (see Eq. (20) and Eq. (28) of Janssen (2003)). Mori \& Janssen (2006) additionally invoked the assumption of narrow bandwidth, which is consistent with the cubic Nonlinear Schrödinger equation (NLS), and assume an underlying one-dimensional Gaussian spectrum.

For unidirectional waves, the result by Mori \& Janssen (2006) implies an excess kurtosis that increases with a time scale dependent on the bandwidth of the initial spectrum and the dominant wave period (Janssen \& Bidlot 2009; Mori et al. 2011). Following monotonic increase from zero, the dynamic excess kurtosis then levels off tending to a value of $\pi /(3 \sqrt{3})$ times the Benjamin-Feir Index (BFI) squared, where the BFI is a measure of the ratio of the significant wave steepness to the bandwidth of the waves (Mori \& Janssen 2006). Fedele (2014) obtained an equation for the evolution of excess kurtosis using the one-dimensional (1D) cDZ equation of Fedele \& Dutykh (2012), based on Dyachenko \& Zakharov (2011). The cDZ equation is valid for weakly nonlinear four-wave interactions like the NLS, but does not have any constraints on the spectral bandwidth unlike the NLS (Fedele 2014). The excess kurtosis based on the cDZ equation is generally less than predicted by the equivalent NLS expression, and the reduction is greater for greater bandwidth (see Eq. (D16) of Fedele (2014)).

It is important to distinguish conceptually, on the one hand random wave fields that are homogeneous in space and evolving and thus non-stationary in time and on the other hand those that are stationary in time and evolving and thus in-homogeneous in space. The aforementioned authors (Janssen (2003), Mori \& Janssen (2006), Janssen \& Bidlot (2009) and Mori et al. (2011) and Fedele (2014)) all examined the evolution of spatially homogeneous fields in time. Using analogous premises to Mori \& Janssen (2006), an equation for the spatial evolution of temporally homogeneous waves has been derived by Fedele et al. (2010). In the narrow-banded limit, the results of Mori \& Janssen (2006) and Fedele et al. (2010) are equivalent, and mapping between space and time takes place using the group velocity (see also Chabchoub \& Grimshaw (2016)), noting that the bandwidth in frequency is half the bandwidth in wavenumber (Fedele et al. 2010). For the broad-banded cDZ, mapping between space and time is not straightforward, and we are unaware of any authors describing the evolution of kurtosis in space for temporally homogeneous waves based on the cDZ, although a spatial of the Zakharov equation exists (Shemer et al. 2001; Kit \& Shemer 2002).

Laboratory measurements are almost exclusively made in the time domain at a finite number of wave gauges (Fedele et al. 2010; Zhang et al. 2014, 2016; Onorato et al. 2004, 2006; Kokorina \& Slunyaev 2019). Apart from the time-domain experiments, spatiotemporal measurements are beginning to be successfully made in the laboratory using stereo-imaging techniques (Zavadsky et al. 2017) commonly applied in the field (e.g. Fedele et al. (2013)). A restriction of many of the validation studies in the laboratory is the length of the experiments, meaning that only the initial stages of the evolution have been compared to theory. We focus herein on the laboratory experiments of Onorato et al. (2004) (henceforth O04), which were compared to the theoretical results of Mori \& 
Janssen (2006) by Mori et al. (2007) and to numerical simulations of the Dysthe equation (Dysthe 1979) by Onorato et al. (2005). In doing so, we consider evolution of temporally homogeneous (or stationary) waves in space.

Previously, Shemer \& Sergeeva (2009) presented experimental results for the spatial evolution of wave statistics along the wave tank for unidirectional random waves, and their observed short-term probability distributions were well predicted by the third-order model of (Tayfun \& Fedele 2007). Shemer et al. (2010b) compared these experimental results with the cubic NLS and the MNLS, and MNLS could provide satisfactory predictions of individual groups in the time domain as well as statistical parameters. Shemer et al. (2010a) further examined the impact of the initial spectral width on the evolution of the wave spectrum, and wave statistics. Slunyaev \& Sergeeva (2012) examined the phase correlation during the initial stage of evolution and its connection with the evolution of wave statistics.

In nature, storm waves are directionally spread, and this makes a fundamental difference to the nonlinear physics. Janssen \& Bidlot (2009) first showed that kurtosis is generally reduced by directional spreading. Extending the results by Mori \& Janssen (2006) based on the unidirectional NLS, Fedele (2015) then showed analytically that the normalised excess kurtosis of directionally spread waves reaches a maximum and eventually tends monotonically to zero as the wave field reaches a quasi-equilibrium, finding good agreement with the experimental data of Onorato et al. (2009) and numerical simulations by Toffoli et al. (2010) (see also Xiao et al. (2013); Annenkov \& Shrira (2009)). Based on the 2D+1 NLS, the result for directionally spread waves by Fedele (2015) thus paints a very different picture with large values of kurtosis and associated rogue waves being transient (see Janssen \& Janssen (2019) for further discussion of the asymptotics).

In this paper, we perform both new laboratory experiments and numerical simulations to examine how the kurtosis of unidirectional water waves evolves over relatively long distances. We compare our experimental and numerical results with the experiments of O04 and with the theoretical solutions for the evolution of the kurtosis of unidirectional random waves based on the NLS by Mori \& Janssen (2006) and based on the cDZ by Fedele (2014). Throughout, we cite Mori \& Janssen (2006), although full details of the evolution are developed in Janssen \& Bidlot (2009) and Mori et al. (2011) and we actually compare to the closed-form solution given in Fedele et al. (2010) (see appendix A). These theoretical solutions, with the exception of Fedele et al. (2010), are valid for waves evolving in time, and we convert these to waves evolving in space using relationships only valid in the narrow-banded limit (Fedele et al. 2010). Our numerical simulations are performed using two methods: OceanWave3D (Engsig-Karup et al. 2009), which solves the fully nonlinear (potential-flow) water wave equations, and the Modified Nonlinear Schrödinger (MNLS) model of Trulsen et al. (2000).

For the steep cases, both our experiments and our numerical simulations (using both methods) show that the kurtosis peaks before returning to an equilibrium level. Such transient maxima are not predicted by the NLS-based and cDZ-based analytical models of Mori \& Janssen (2006) and Fedele (2014). We note that these transient maxima in kurtosis can also be observed in the experiments of $\mathrm{O} 04$ and analogous simulations of the Dysthe equation (Dysthe 1979) by Onorato et al. (2005), where maxima are noted but not explored in detail. Peaks in kurtosis are also found in numerical simulations of the NLS by Onorato et al. (2016), where the evolution of kurtosis is linked to the evolution of the spectral bandwidth. Using the MNLS, we study how the properties of these transient maxima for unidirectional waves depend on input steepness and bandwidth. We note that this pattern of the kurtosis reaching a peak is superficially similar to what is predicted and observed for directionally spread behaviour (Onorato et al. 2009; Toffoli et al. 2010; Fedele 
2015), except there the kurtosis is predicted to return to zero at long distances (Janssen \& Janssen 2019), although this may not always be observed (Xiao et al. 2013). We also examine how the expected shape of an extreme wave group evolves over the same distance. To our knowledge, this has not previously been examined for this problem. We find significant asymmetry during the initial evolution. Locations of maxima in asymmetry coincide approximately with the locations of the maxima in kurtosis. We emphasise our paper is confined to unidirectional waves, so that its findings cannot readily be extended to realistic ocean waves. Nevertheless, investigating unidirectional waves as a limiting case of directionally spread waves is useful for three reasons. First, it will help elucidate the nonlinear physics at work, especially over longer distances. Second, it will inform offshore engineering models tests, which are still often conducted in unidirectional waves. Third, some extreme events can be quite similar in behaviour to the unidirectional limit (e.g. Adcock et al. (2015)). Furthermore, there are analogues between 1D waves and those in other media such as optical fibres (Dudley et al. 2019).

\section{Methods}

\subsection{Experimental set-up}

The experiments were carried out in the Multifunction Towing Tank at Shanghai Jiao Tong University. The tank is $300 \mathrm{~m}$ by $16 \mathrm{~m}$ and has a flat bed with a water depth of $7.5 \mathrm{~m}$, giving a non-dimensional water depth for our experiments of $k_{0} d=13.4$ based on the spectral peak. There are 40 hinged-flap type wavemakers at one end of the flume. Linear wave generation theory was applied, and the impact of second-order error waves on the overall wave statistics was analysed carefully and found not to significantly affect the results. There was a parabolic beach at the far end of the flume opposite the wavemakers. Reflection analysis suggests that less than $3 \%$ of the energy is reflected. The wave surface elevation was measured by 10 capacitance probes at $100 \mathrm{~Hz}$ with excellent calibration characteristics. However, the wave probes could only be installed on a movable carriage. To track the wave evolution over a wider range, the experiments were repeated with different carriage positions. Irregular wave repeatability tests showed very consistent wave statistics at the same position over five repeats.

\subsection{Numerical methods}

We use two numerical modelling approaches in this paper. First, we solve the fully nonlinear potential flow equations for water waves using OceanWave3D (Engsig-Karup et al. 2009). The numerical wave tank length in the wave propagation direction is 778 $\mathrm{m}$, which we discretise with 10242 nodes giving a spatial resolution of $0.076 \mathrm{~m}$. The water depth of the wave tank is $7.5 \mathrm{~m}$ covered by 15 clustered nodes. Care has been taken, following the approach of Barratt et al. (2020), to ensure sufficient resolution to accurately capture the nonlinear physics. The simulation time is $1920 \mathrm{~s}$ - identical to the experiments. Waves are generated using a relaxation zone at the start of the domain and are absorbed by a damping zone at the end of the flume. A wave breaking model is applied, which is triggered by downward Lagrangian particle accelerations on the free surface that are larger than $0.4 \mathrm{~g}$. After determining these breaking events, a filter is applied to the local free surface region to remove energy from the waves until the downward particle accelerations are below the threshold.

We also use a faster code which solves the Modified Nonlinear Schrödinger (MNLS) model of Trulsen et al. (2000). Using this MNLS model, which unlike our fully nonlinear model does not capture wave breaking, should give us confidence that the physics we 
are observing is not influenced by wave breaking. The fast computation times also mean that the MNLS results can be run repeatedly, reducing the uncertainty in the estimated kurtosis sufficiently that confidence bands associated with statistical variability are not required. The model set-up is similar to that above with waves generated using a relaxation zone and absorbed at the far end of the domain. We use a very high (for an envelope model) spatial discretion of 23 points per wavelength. In addition to standard numerical checks, we can also test this code for energy conservation by studying the related problem of the evolution of a 'sea state' covering the entire domain with wraparound boundary conditions at the end. For this problem with the same initial spectrum, energy loss over 100 wave periods is less than $0.5 \%$.

\section{Results: kurtosis}

In this section, we first compare the experimental results and numerical simulations with both analytical results for the evolution of normalised dynamic excess kurtosis based on the NLS (Mori \& Janssen 2006) and the cDZ (Fedele 2014) equations and the experiments of O04. To broaden the parameter space, we further examine a range of input 'sea-state' parameters using the MNLS with Gaussian input spectra.

\subsection{Comparison with the experiments of 004}

\subsection{1. 'Sea-state' parameters}

We choose to study cases based on the seminal experiments of O04. These are summarised in table 1 . The cases are based on the JONSWAP spectrum with different peak enhancement factors. The Benjamin-Feir Index (BFI) is computed following the method recommended in Serio et al. (2005), who base their recommendation on a review of different methods:

$$
\mathrm{BFI}=\sqrt{m_{0}} k_{0} Q_{p} \sqrt{2 \pi}
$$

where $m_{0}=H_{s}^{2} / 16$ is the zeroth moment of the energy spectrum with $H_{s}$ the significant wave height, $k_{0}$ is the peak wavenumber, and $Q_{p}$ is a dimensionless parameter that describes the spectral bandwidth (Goda 2000). The parameter $Q_{p}$ has less sensitivity to the high-frequency tail of the spectrum (and cut-off frequency) than other bandwidth metrics (Serio et al. 2005), and is given by:

$$
Q_{p}=\frac{2}{m_{0}^{2}} \int_{0}^{\infty} f S^{2}(f) \mathrm{d} f
$$

where $S(f)$ is the variance density spectrum. We note that the BFI is not the most robust numerical parameter, as its precise value is strongly dependent on the way the bandwidth is calculated. The waves we generated during the experiment were slightly steeper and considerably more narrow-banded than those in the experiments of O04 (see table 1). For completeness, we report in table 1 the values of the parameters corresponding to our experiments, to the experiments of $\mathrm{O} 04$ calculated with the method used in our paper (in round brackets) and to the experiments of $\mathrm{O} 04$ as reported in its table 1 [in square brackets]. We emphasise the considerably higher values of BFI we obtain in comparison those reported in table 1 of O04. Whilst the waves made numerically agree very well in terms of the spectral shape with those desired (the input conditions in O04), we found the waves created experimentally to be somewhat larger in terms of significant wave height and considerably more narrow-banded (see table 1). This should be allowed for in the comparisons that follow. 
TABLE 1. 'Sea-state' parameters of the three test cases measured at the first probe, with $T_{0}$ the peak period, $\nu=\sqrt{m_{0} m_{2} / m_{1}^{2}-1}$ the bandwidth parameter, where $m_{n}$ are $n$ th-order spectral moments of the variance density spectrum $S(\omega)$ in angular frequency $\omega$. Also shown are the 'sea-state' parameters of the experiments of O04 calculated with the method used herein (in round brackets) and the 'sea-state' parameters of the experiments of O04 as reported in their table 1 [in square brackets].

\begin{tabular}{ccccccc}
\hline Case & $\gamma$ & $H_{s}[\mathrm{~m}]$ & $\varepsilon=k_{0} H_{s} / 2$ & $\nu$ & $Q_{p}$ & BFI \\
\hline 1 & 1.0 & $0.125(0.116)[0.11]$ & $0.113(0.103)[0.098]$ & $0.14(0.15)$ & $3.87(3.88)$ & $0.6(0.5)[0.2]$ \\
2 & 3.3 & $0.162(0.143)[0.14]$ & $0.144(0.129)[0.125]$ & $0.09(0.13)$ & $6.71(5.92)$ & $1.3(0.9)[0.9]$ \\
3 & 6.0 & $0.182(0.168)[0.16]$ & $0.160(0.150)[0.142]$ & $0.08(0.12)$ & $8.68(7.69)$ & $1.9(1.3)[1.2]$ \\
\hline
\end{tabular}
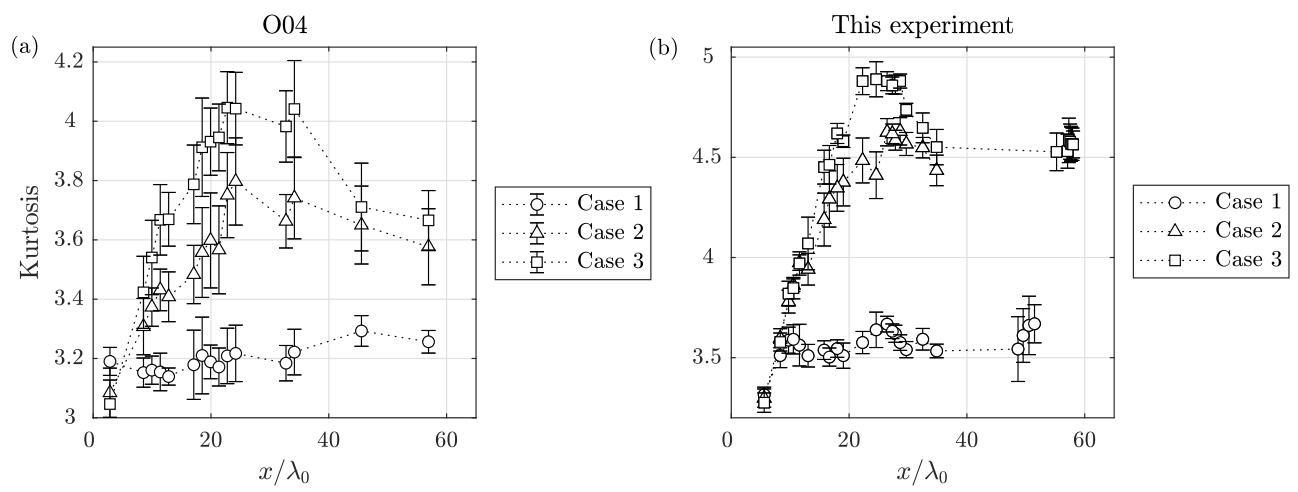

Figure 1. Spatial evolution of kurtosis: (a) results from Onorato et al. (2004), (b) our results. Error bars show the $95 \%$ of confidence interval based on standard deviation. The distance from the wavemaker is denoted by $x$, and $\lambda_{0}$ is the peak wave length.

\subsubsection{Spatial evolution of kurtosis}

We start by comparing our experimental results with those of O04. Figure 1 shows the evolution of kurtosis for the three cases. Despite the slight mismatch in the size of the waves, there is good basic agreement in the shape of the curves between the different experiments. With more probes positioned around the kurtosis peak, we are able to provide a better insight of where the kurtosis reaches its peak, which is most clearly observable for case 3 .

Figure 2 presents the spatial evolution of the normalised excess kurtosis for the three cases using the different approaches (experiments, OceanWave3D and MNLS). The excess kurtosis, $C_{4}^{d}$, is corrected for the presence of bound waves using the method of Tayfun (1980). We have ensemble averaged the value of excess kurtosis to provide a clearer overall trend. The fluctuations that remain, especially for the experiments and OceanWave3D simulations, are the result of a relatively small number of ensemble members owing to their significant (computational) costs. Only 5 ensemble members were used for the experiments and 9 for the OceanWave3D simulations. Hence, we have added confidence intervals for the experimental and OceanWave3D results. Both numerics and experiments show the same general trends. In the simulations presented, the MNLS captures the overall trend in all cases. Figure 2 also shows the theoretically predicted solution of excess kurtosis of Mori \& Janssen (2006) based on the NLS and Fedele (2014) based on the cDZ (see appendix A for the exact equations we use). Both the theoretical predictions assume Gaussian input spectrum, which is different from the JONSWAP spectrum for 


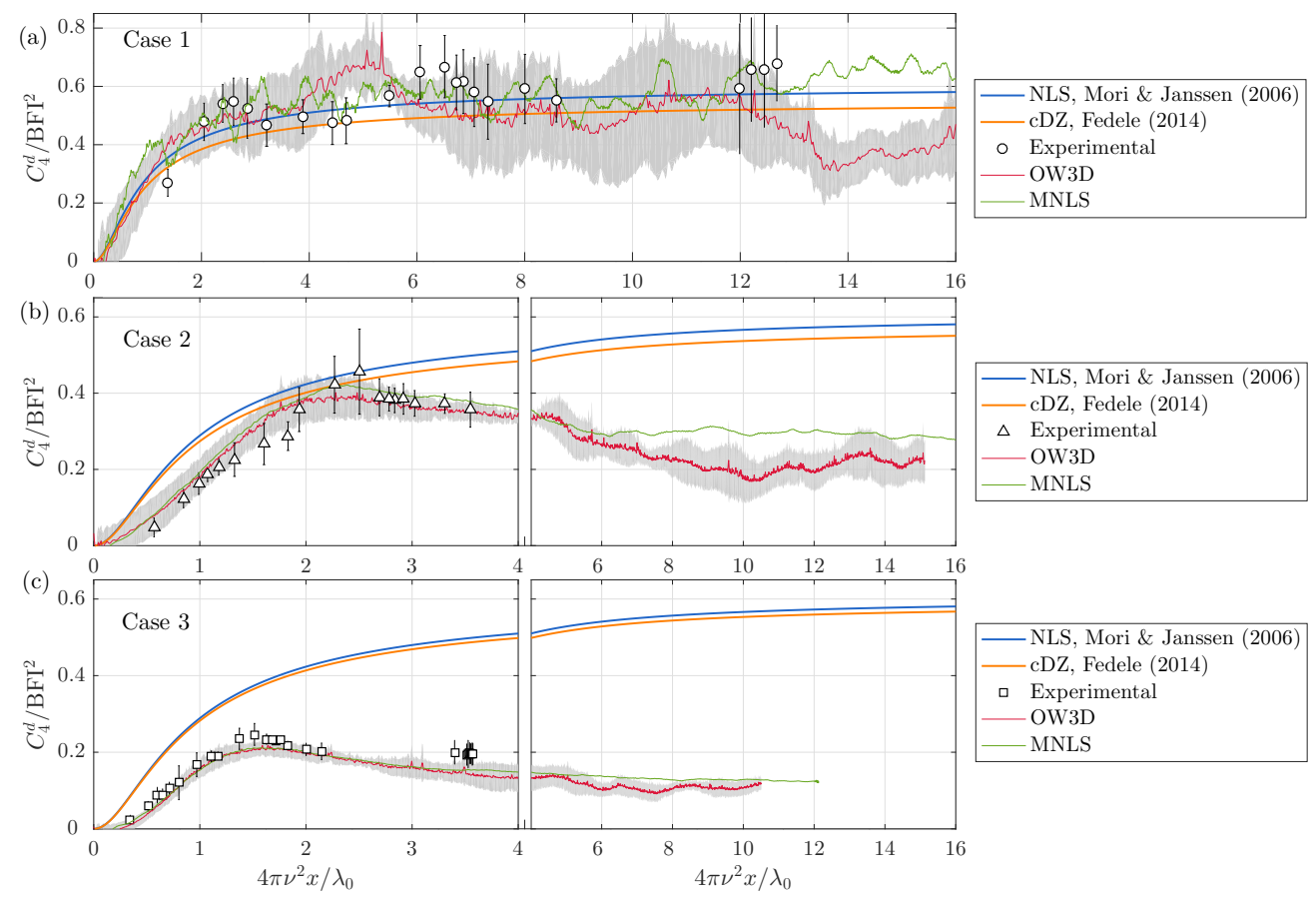

FIGURE 2. Evolution of normalised dynamic excess kurtosis at different distances from the wave generator: (a) case 1, (b) case 2 and (c) case 3 . Shading represents the $95 \%$ confidence intervals for OceanWave3D simulation with 8 different random seeds. A total of 120 different random seeds are used in MNLS simulations. Consequently, for the MNLS, the error bars are negligible and have been omitted for clarity. The parameter $C_{4}^{d}$ is dynamic excess kurtosis, BFI is the Benjamin-Feir Index, $\nu$ is the input bandwidth and $\lambda_{0}$ is the peak wave length.

experiments and simulations. It is worth mentioning that both solutions are given for spatially homogeneous waves evolving in the time domain in the original papers. We convert these to the temporally homogeneous waves evolving in space we study using the group velocity, which is valid in the context of the already narrow-bandwidth restricted NLS (Fedele et al. 2010) but only valid in the narrow-bandwidth limit of the cDZ. What we label cDZ in figure 2 is only the leading-order correction for broad bandwidth from Fedele (2014) (see appendix A).

The agreement of the two theoretical solutions, which give comparable predictions, with the experiments is very good over the whole course of the evolution for case 1 (the least steep and most broad-banded case). The experiments and theoretically predicted solutions start with an excess kurtosis of zero for all cases. Asymptotic analysis (see Janssen \& Bidlot (2009) and §3.2) of the analytical solution based on the NLS suggests that the initial growth rate of kurtosis should be quadratic in space. In cases 2 and 3 (the steeper cases), there appears to be a delay in the onset of the kurtosis increasing in the experiments compared to both theoretical solutions, as also observed in Fedele et al. (2010). After different non-dimensional length scales, cases 2 and 3 both depart entirely from the theoretical solutions. Both cases peak without exceeding the theoretical value. The excess kurtosis then slowly decreases until it reaches a steady-state value. Before we interpret the results in figure 2, we note that they depend strongly on the initial value of $\mathrm{BFI}$, since its square acts as the normalising factor on the vertical axis. We also present a version of this figure in which excess kurtosis is normalised by steady-state BFI in appendix B. 
To interpret these results, we note that the theoretical solutions based on the NLS and the cDZ are based on small-steepness and narrow-bandwidth approximations (with the cDZ accounting for a wider bandwidth than the NLS, cf. appendix A). Thus, we would expect these solutions to work best in the region where the approximation is most valid, i.e. for the lower steepness waves with narrower bandwidths. Taking into account the leading-order balance between these two effects, the theoretical solution should work best when their ratio, as captured by the BFI, is smallest. This is consistent with our finding in the present paper, since the theory works best for the low-BFI case where the bandwidth of the spectrum is largest. Although the cDZ does explain a small reduction in kurtosis predictions compared to the NLS, it does not predict a maximum.

Hypothesising what happens at the level of a wave group, a spatial contraction of the wave group takes place around a large wave, which can be thought of as a local expansion of bandwidth. This effect is dependent on the Benjamin-Feir index of the 'sea state' (see for instance Eq. (3.12) in Adcock \& Taylor (2009)). It is important when considering the limitations of NLS-type equations not to base the bandwidth limitation on the input spectrum but on the local extremes which occur within the simulation.

\subsubsection{Spatial evolution of the spectrum}

Figure 3 shows the spatial evolution of the spectrum for case 3 . It can be seen that all the spectral change takes place during the initial phase of the simulation before the kurtosis reaches its peak (see also Shemer et al. (2010a)).

To examine this further, we consider what happens if, during the evolution, we randomise the phase of the simulations removing all the correlations between components. We do this only using the MNLS as phase randomisation is more straightforward when bound waves are not directly simulated. Figure 4 presents the results of these simulations. Where phase randomisation occurs, the excess kurtosis is zero (i.e. at $x / \lambda_{0}=168$, 380 ). The energy of the system is kept as constant at each randomisation. Following the randomisation, the kurtosis increases rapidly although over a slightly longer length scale at each randomisation, presumably due to the slight broader spectrum at each successive randomisation. After each successive randomisation, the kurtosis reaches a peak in kurtosis before slowly settling back to a steady-state value. We find that after each randomisation the maximum value and the steady-state value are slightly lower. This demonstrates that prescribed uncorrelated initial random phase distribution is different from the correlated phase distribution at steady state as a result of nonlinear evolution (see also Slunyaev \& Sergeeva (2012)). Comparing figure 4 (a) and (b), it is evident that the peaks in kurtosis go hand in hand with an overshoot in the broadening of the spectrum.

\subsubsection{Wave breaking}

In the above discussion, we have ignored the effect of wave breaking. Wave breaking is modelled in the fully nonlinear simulations and is, of course, present in the experiments. Wave breaking is most active in the steepest and most nonlinear cases. Although this may play some role in our results, we suspect that breaking is of relatively minor importance, as our MNLS results, which do not include breaking, show very similar general results. Of course, over very long distances wave breaking will dissipate energy from the system. This takes place on a length scale much greater than we have considered.

\subsection{Gaussian input spectra}

To explore the parameter space further, we consider a second set of simulations where we just utilise the MNLS model. We do this to explore a significantly wider parameter 


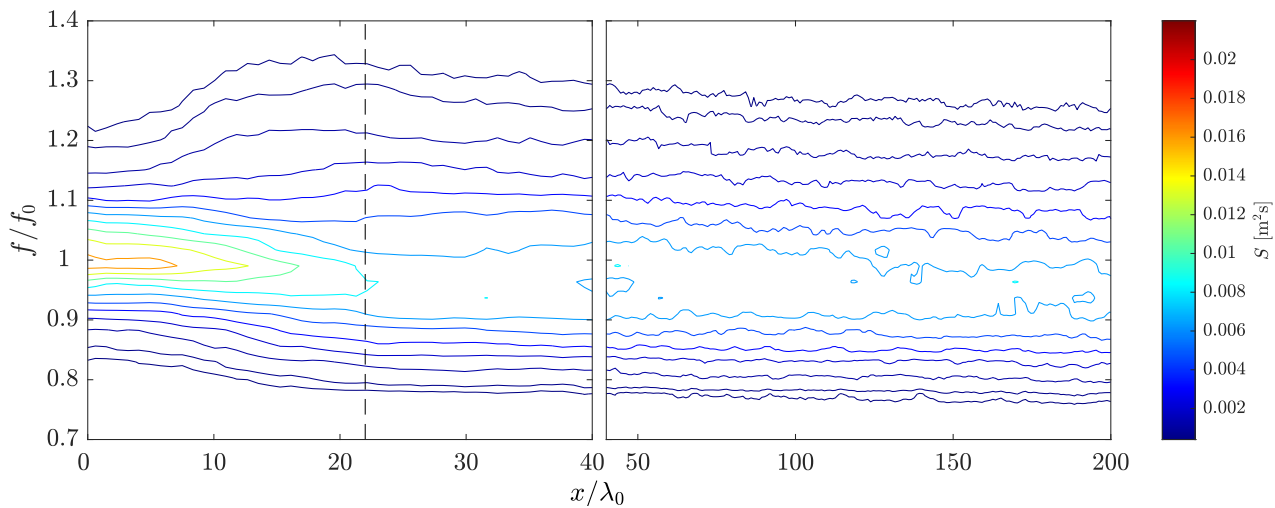

FiguRE 3. Spatial evolution of the frequency spectrum of case 3 averaging over multiple fully nonlinear simulations using OceanWave3D. The dashed vertical line shows the location of peak kurtosis.

(a)
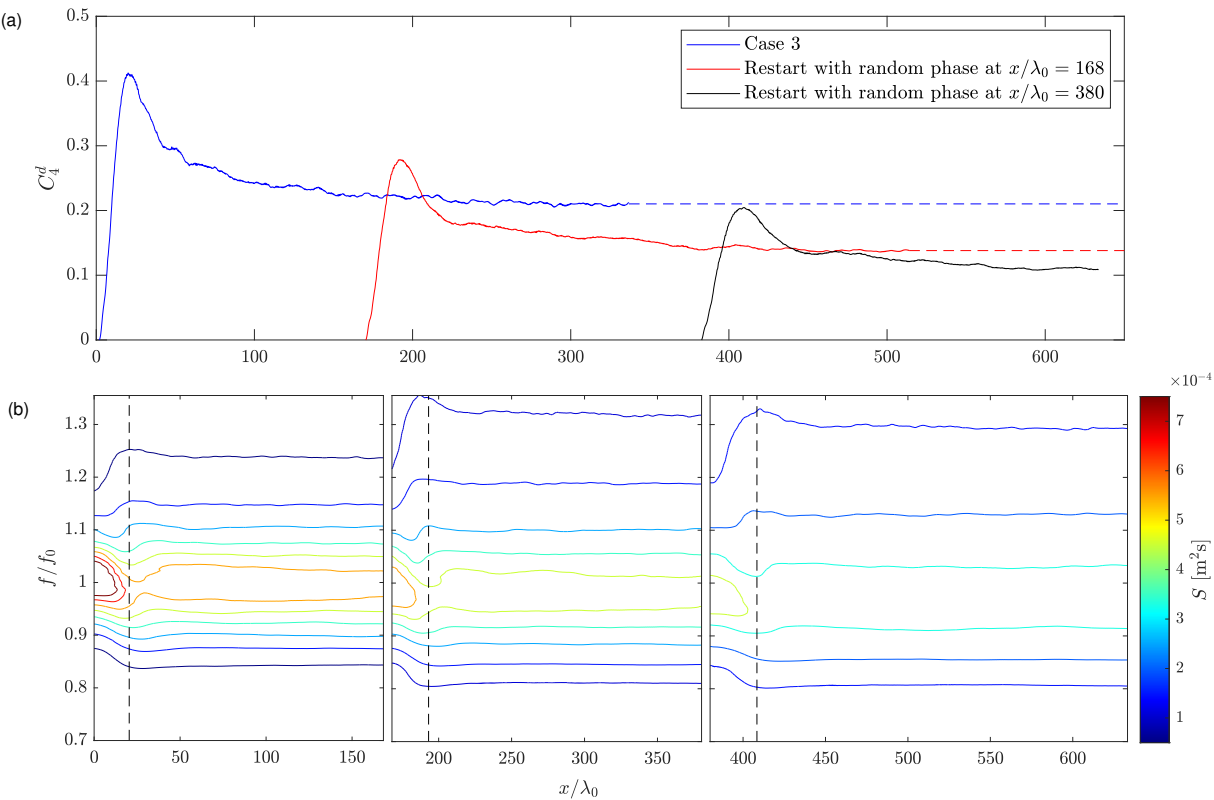

FIGURE 4. (a): Spatial evolution of the dynamic excess kurtosis for case 3 with phases randomised at $x / \lambda_{0}=168,380$. The dashed lines indicate the steady-state excess kurtosis value. (b): Corresponding spectral evolution at different locations. The dashed vertical line shows the location of peak kurtosis.

space. We use Gaussian input spectra as it is more straightforward to define a bandwidth for such spectra than for spectra with an algebraic frequency tail, such as the JONSWAP spectrum used in the experiments of O04. Furthermore, the theoretical solutions of Mori \& Janssen (2006) assume a narrow-banded Gaussian input spectrum. The Gaussian input spectrum is defined as:

$$
S(k)=\left(\frac{H_{s}}{4}\right)^{2} \frac{1}{\Delta f \sqrt{2 \pi}} \exp \left(-\left(f-f_{0}\right)^{2} /\left(2 \Delta f^{2}\right)\right),
$$


where $f_{0}$ is the peak frequency and $\Delta f$ controls the bandwidth of the spectra. We vary input steepness and bandwidth, but note our test matrix is not uniformly spaced in steepness and bandwidth. This irregular spacing was to explore as wide a range of values as possible without running cases that were too nonlinear or would take very long distances to reach a steady state. Since the leading-order broad-bandwidth correction from the cDZ (Fedele 2014) is small, we only compare to NLS-based (Mori \& Janssen 2006) theory here.

We will start by considering the initial rate of increase of the kurtosis. For spatially homogeneous waves that evolve in time, Janssen \& Bidlot (2009) showed that the theoretical solutions of Mori \& Janssen (2006) reduce to a quadratic initial evolution of normalised excess kurtosis with respect to time (see also Mori et al. (2011) and Fedele (2015)). Noting that for these narrow-banded spectra evolution in time can simply be expressed as evolution in space using the group velocity (Fedele et al. 2010), this quadratic evolution in time corresponds to the following initial quadratic evolution in space of normalised excess kurtosis, $C_{d}^{4} / \mathrm{BFI}^{2}$, for the temporally homogeneous, unidirectional waves we study:

$$
\frac{C_{d}^{4}}{\mathrm{BFI}^{2}}=\frac{1}{2} \xi^{2} \quad \text { for } \xi \ll 1,
$$

where the intrinsic dimensionless length $\xi \equiv 4 \pi \nu^{2} x / \lambda_{0}, x$ the dimensional length, and $\lambda_{0}$ the peak wave length. If we express $\xi$ as the ratio of distance from the wavemaker $x$ and what we call the initial growth length scale $L_{s}$, we can express (3.4) as $C_{d}^{4} / \mathrm{BFI}^{2}=$ $(1 / 2)\left(x / L_{s}\right)^{2}$. We can now compare the theoretical prediction of the length scale $L_{s \xi} \equiv$ $\lambda_{0} /\left(4 \pi \nu^{2}\right)$ to an estimate from our data, which we obtain from the inverse of the rate of change of the square root of normalised excess kurtosis for $\xi<0.5$ :

$$
L_{s}=\left(\frac{\mathrm{d}}{\mathrm{d} x} \sqrt{\frac{2 C_{d}^{4}}{\mathrm{BFI}^{2}}}\right)^{-1} \quad \text { for } x \ll \frac{\lambda_{0}}{4 \pi \nu^{2}} .
$$

Figure 5a presents the comparison of our estimate of the initial growth length scale from simulations of the MNLS $L_{s}$ to its theoretically predicted counterpart $L_{s \xi}$. For the most narrow-banded cases, the growth length scale is quite close to the theoretical values. However, theory seems to over-predict the initial growth length scale for broad-banded cases. Steepness has only a small role.

Figure $5 \mathrm{~b}$ shows the normalised peak kurtosis $\left.C_{d}^{4}\right|_{\max } / \mathrm{BFI}^{2}$ reached during each simulation. The NLS-based theory of Mori \& Janssen (2006) predicts the steady-state value to be at $\pi /(3 \sqrt{3}) \sim 0.604$. This is close to the value reached by the least nonlinear case we considered. However, as either steepness increases, or bandwidth decreases, the peak value of kurtosis normalised by the value of the Benjamin-Feir Index squared decreases. This behaviour, and the discussion of the physical reasons for it, is consistent with that presented above for the cases based on the JONSWAP spectrum.

Finally, we consider the steady-state value that is reached at the end of the simulations. Reaching a steady state takes a different distance for different simulations. We determine steady-state kurtosis $\left.C_{d}^{4}\right|_{\text {ss }}$ as the averaged kurtosis over a distance of $\Delta x / \lambda_{0}=50$, where the maximum variation in the excess kurtosis over this distance is less than $10 \%$ of the maximum excess kurtosis. Figure 5c presents the steady-state value of normalised steadystate kurtosis for a range of input conditions. The trends are very similar to those for peak kurtosis. Essentially, the higher the starting BFI, which results in a larger denominator for the normalised kurtosis, the smaller is the steady-state normalised kurtosis.

Additional insight into the steady-state values of kurtosis is given by considering 

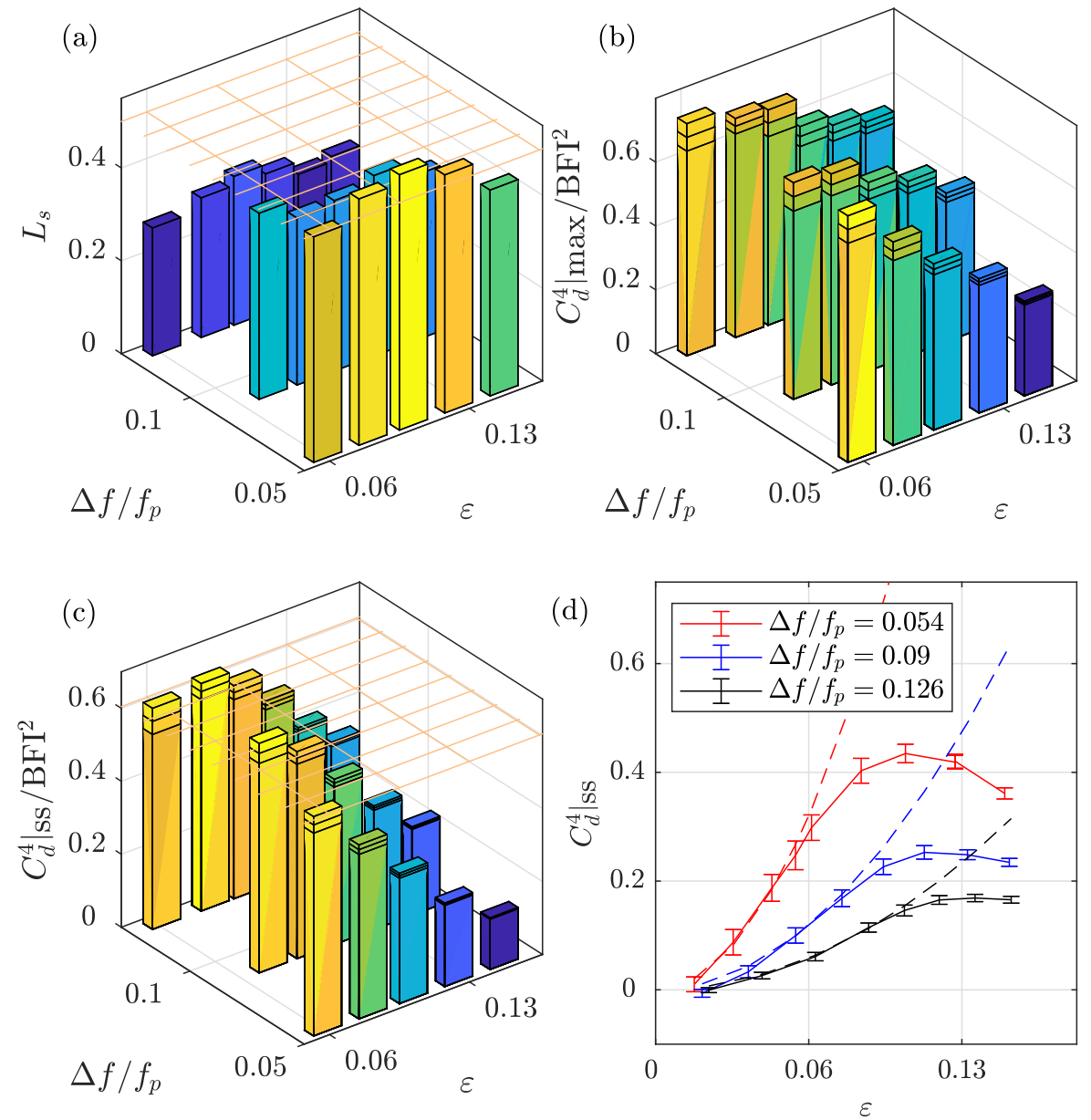

FiguRE 5. Analysis of kurtosis of random waves from an initially Gaussian spectrum: (a) ratio between the initial growth length scales of normalised excess kurtosis predicted by the simulation $L_{s}$ and theory $L_{s \xi}$, (b) maximum value of kurtosis $\left.C_{d}^{4}\right|_{\max }$ reached during the simulation, (c) steady-state value of normalised excess kurtosis $\left.C_{d}^{4}\right|_{\mathrm{ss}} / \mathrm{BFI}^{2},(\mathrm{~d})$ steady-state value of kurtosis $\left.C_{d}^{4}\right|_{\text {ss }}$ as a function of the initial steepness $(\varepsilon)$ for three different initial bandwidths $\left(\Delta f / f_{0}\right)$. The grid in (a), (c) and the dashed lines in (d) shows the theoretical prediction based on Mori \& Janssen (2006). The error bars in (b), (c) and (d) show 95\% confidence interval based on the standard deviation.

steady-state kurtosis without normalisation for cases with different steepnesses but the same bandwidths. Figure 5d shows the results for three different bandwidths, where we have also added the theoretical predictions based on Mori \& Janssen (2006). For sufficiently small steepness, we observe an increase in the steady-state kurtosis with steepness, as expected. The values of kurtosis measured from simulations agree well with the theoretical predictions. However, above a certain initial steepness, which depends on the initial bandwidth, the steady-state kurtosis appears to flatten off as steepness is increased further. This flattening also causes the measured kurtosis at steady state to depart from the theoretical predictions. This figure further demonstrates the discrepancy between numerical simulations and theoretical predictions for the steady-state values of normalised kurtosis in figure 2 . 


\section{Results: group shape}

In a linear model of wave evolution, the theory of quasi-determinism can be used to describe the shape of extreme waves (Lindgren 1970; Boccotti 2000; Tromans et al. 1991). As part of this theory, the average shape of an extreme crest is given by the scaled auto-correlation function (Boccotti 1983) and the shape of the wave with the largest crestto-trough height by the scaled difference of two time-shifted auto-correlation functions (Boccotti 1989). In the time domain, the expected shape of an extreme crest $\eta=\eta_{\max }$ at any location $x$ (with the largest crest shifted to occur at $t=0$ ) is given by:

$$
\eta(t, x)=\frac{\eta_{\max }}{m_{0}} \int_{0}^{\infty} S(f, x) \cos (2 \pi f t) \mathrm{d} f
$$

where $S(f, x)$ is the power spectral density function at the location of the measurement $x, m_{0}=H_{s}^{2} / 16$ is the zeroth moment of the energy spectrum at that location. Nonlinear physics might be expected to modify this. For deep-water waves, the effect of nonlinear physics on the shape of a nonlinear event has mainly been studied for wave groups (e.g. Baldock et al. (1996)). For a unidirectional wave group, analytical results based on the NLS predict that the group would contract spatially, and this is dependent on the amplitude-to-width ratio of the group (analogous to the BFI but for a group) (Adcock \& Taylor 2009). Little attention has been paid to unidirectional random waves with the study of Lo \& Mei (1985) and the recent work of Dematteis et al. (2019) being exceptions. In this section, we consider the shape (in the time-domain) of extreme events throughout the spatial evolution using the MNLS with input Gaussian spectra.

Figure 6 (a) presents the measured average shape of the 20 largest crests at different locations in the numerical tank. The bandwidths at the different locations are $\nu=$ $\{0.12,0.15,0.18,0.18,0.18,0.17\}$ corresponding to $x / \lambda_{0}=\{0,10,20,30,40,50\}$. Figure 6 (b) presents the linear predictions of these events at the same position based on the theory of quasi-determinism. The second-order bound harmonics are excluded in the figure, as the MNLS code computes the free wave directly without second-order bound harmonics. When generated, the group is symmetric and consistent with linear theory. As the waves evolve in space, the shape of the wave groups is modified by nonlinear physics. The main characteristics of this modification are a movement to the front of the wave group of the largest wave and a contraction of the wave group. We have also presented the shape of the crests profiles predicted by the theory of quasi-determinism shown in figure 6 (b). The changes in the predicted profiles in figure 6 (b) are only due to changes in the wave spectrum with $x$ (cf. $(S(f, x)$ in (4.1)). Additionally, we present the averaged shape of the wave with the largest crest-to-trough height in appendix $\mathrm{C}$.

We quantify the changes to group shape using two parameters (following Tang et al. (2019)). The first is a measure of the width of the group and the second a measure of the asymmetry. We begin by defining $\sigma_{1}$ and $\sigma_{2}$ as the durations when the envelope height exceeds $80 \%$ of the peak height of the envelope obtained by averaging the envelope corresponding to the 20 largest waves, where the envelope is obtained using a Hilbert transform. As illustrated in figure $7, \sigma_{1}$ denotes the duration of the front flank of the group (passing the observer at earlier time) and $\sigma_{2}$ the duration of the rear flank (passing the observer at later time). We now define the parameters $B_{1}$ and $B_{2}$ to be the durations $\sigma_{1}$ and $\sigma_{2}$ as fractions of what would be predicted by linear theory from the local spectrum (i.e. from the theory of quasi-determinism):

$$
B_{1}=\frac{\sigma_{1, \text { measured }}}{\sigma_{1, \text { quasi-determinism }}}, \quad B_{2}=\frac{\sigma_{2, \text { measured }}}{\sigma_{2, \text { quasi-determinism }}} .
$$

The ratios $B_{1}$ and $B_{2}$ quantify the nonlinear modifications to both sides of the measured 
(a)

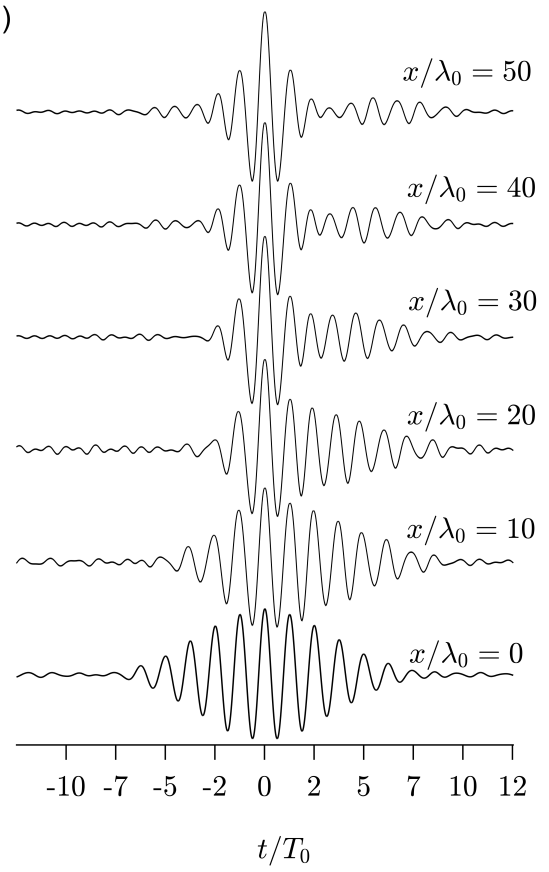

(b)

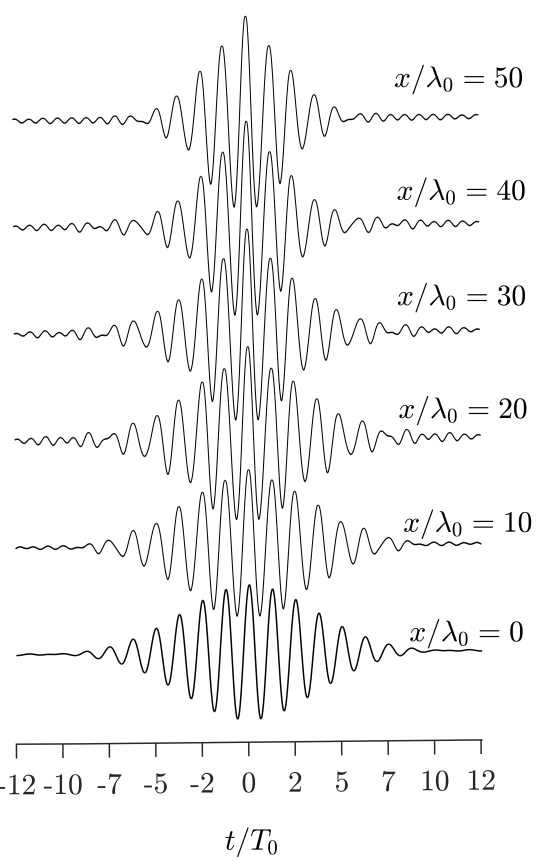

FiguRE 6. Normalised average of: (a) the 20 largest crest profiles out of over 6400 waves, (b) predicted crest profiles based on the theory of quasi-determinism (right) at $x / \lambda_{0}=0,10,20,30,40,50$ for random waves with a Gaussian input spectrum with $\Delta f / f_{0}=0.054$ and $\epsilon=0.044$.

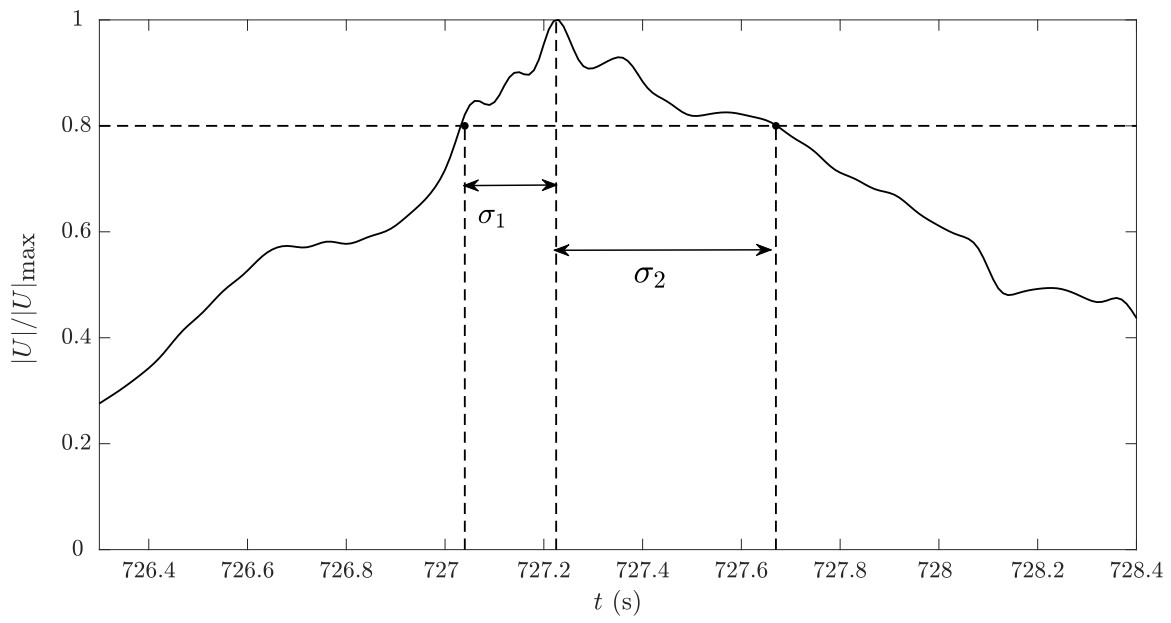

FIGURE 7. Illustration of the envelope duration when the normalised envelope height $|U| /|U|_{\max }$ exceeds $80 \%$ of its peak height.

envelope shape compared to linear theory. We then define $B_{\text {mean }}$ and $\Delta B$ as parameters respectively measuring changes in width and asymmetry relative to linear evolution:

$$
B_{\text {mean }}=\frac{B_{1}+B_{2}}{2}, \quad \Delta B=B_{2}-B_{1} .
$$



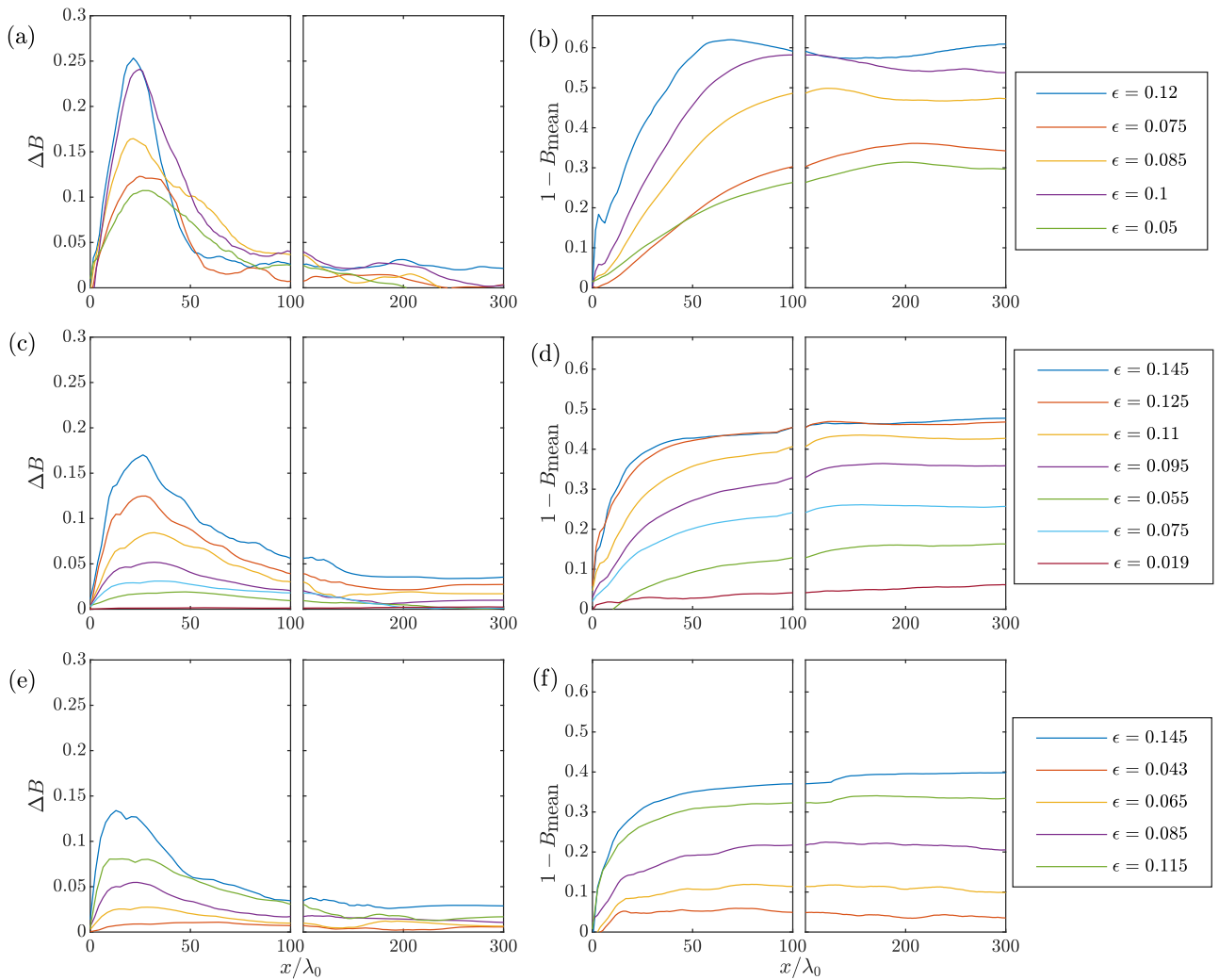

Figure 8. Group shape during nonlinear evolution in space: (a,c,e) envelope asymmetry $(\Delta B)$ and $(b, d, f)$ nonlinear change in the duration when the envelope height exceeds $80 \%$ of its peak height $\left(1-B_{\text {mean }}\right)$ with different initial bandwidths, (a,b) $\Delta f / f_{0}=0.054$ (c,d) $\Delta f / f_{0}=0.09$ and $(\mathrm{e}, \mathrm{f}) \Delta f / f_{0}=0.126$.

Thus, a positive value of $1-B_{\text {mean }}$ implies the group has contracted relative to the shape expected under linear evolution. A positive value of $\Delta B$ implies the largest wave has moved towards the front of the group.

Figure 8 presents the evolution of the parameters for asymmetry and change in group duration. Different initial bandwidth values are given in different panels with different lines for different steepnesses. In all cases, we observe a positive asymmetry, that is, the largest wave moves to the front of the group and arrives earlier. Spatially, the pattern is similar to that of kurtosis. For most cases, there is a peak in the asymmetry parameter during the initial phase before settling down to a smaller near-zero equilibrium value. As for kurtosis, the length scale for equilibrium to be reached is faster for narrower bandwidth. The degree of contraction of the wave-group behaves differently. It increases steadily until it flattens off as equilibrium is achieved. The contraction occurs on a different, much longer, spatial scale than the transient asymmetry.

\section{Discussion and conclusions}

This paper has examined experimentally and numerically how the kurtosis and the shape of large waves, evolves over relatively long distances for unidirectional surface gravity waves. In doing so, we have revisited the seminal unidirectional laboratory 
experiments of Onorato et al. (2004), which we repeat, extend and complement with numerical solutions of the fully nonlinear water wave equations and the MNLS equation of Trulsen et al. (2000). We concentrate on the spatial evolution from a random boundary condition without correlation between components. Following nonlinear evolution, the kurtosis must eventually settle down to a steady state for unidirectional waves (without higher-order nonlinearity), in which changes to the spectrum no longer occur, and the kurtosis has a fixed value greater than that of a Gaussian 'sea state'. We have investigated the transition between these two states and the dependence of the final state on the input conditions.

The picture we find is consistent across the different models and the different cases investigated. For the unidirectional cases studied here, we do not find any significant difference in overall behaviour between JONSWAP and Gaussian spectra, although we note that there is significant ambiguity when calculating the spectral bandwidth for the JONSWAP spectrum. For an input spectrum which is not too narrow-banded or steep (low BFI), theory based on either the NLS (Mori \& Janssen 2006) or cDZ (Fedele \& Dutykh 2012) are excellent models matching both experiments and simulations. However, if the input spectrum is steep or narrow, as captured by a high value of the BFI, the evolution of the excess kurtosis departs from these models. Significant changes occur to the spectrum over relatively short distances. Over the same short distances, excess kurtosis rapidly increases and peaks. The overshoot can be interpreted as being driven by the initial departure of phases from their equilibrium distribution (as can be seen by randomising the phases once steady state has been achieved). Over longer distances excess kurtosis then drops until it reaches a steady-state value with no further evolution of the spectrum. The peak and hence the final value are at a lower kurtosis than that predicted theoretically based on the NLS and cDZ. For cases which are sufficiently nonlinear to depart from the theoretical curve, the kurtosis at steady state appears to be primarily dependent on the initial bandwidth of the spectrum (rather than the steepness).

We note that Janssen (2003) was already aware of the absence of an overshoot in his theoretical calculations based on the kinetic approach; these calculations based on the kinetic approach ultimately led to the result by Mori \& Janssen (2006) we have compared to herein. Janssen's (2003) numerical simulations for the time evolution of spectral width show a similar overshoot to what we have observed for kurtosis (cf. his figure 3). This overshoot is also present in numerical simulations based on the NLS in Onorato et al. (2016) (their figure 1, where the overshoot is also present in bandwidth). This overshoot, Janssen (2003) notes, is likely ignored in his theoretical calculations owing to the assumption that the the action density varies slowly, an assumption which was later relaxed in the Generalized Kinetic Equation of Annenkov \& Shrira (2006). In this paper we have examined a scenario where the spectrum undergoes a transient broadening at the location of the peaks in kurtosis. We thus envisage that calculations based on the Generalized Kinetic Equation of Annenkov \& Shrira (2006) will predict the behaviour of kurtosis for steep and narrow-bandwidth spectra identified herein.

Examining simultaneously the effect of nonlinear evolution on shape of the largest waves, there is a tendency for the largest wave in a packet to move towards the front of the group. This asymmetry follows a similar path to the kurtosis, showing a clear peak during the early stage of evolution before reducing to become relatively small once equilibrium is reached. There is also a reduction in the width of an extreme event making the extreme event appear more transient. This follows a different evolution as it increases monotonically before reaching equilibrium on a longer spatial scale than spatial scale associated with the asymmetry. The locations of the maxima of kurtosis coincide 
approximately with the locations of the maxima of asymmetry of the average shape of extreme events.

We conclude by emphasising our paper is confined to unidirectional waves, so that its findings cannot directly be extended to real-world ocean waves, which are directionally spread. For directionally spread ocean waves, peaks in kurtosis also occur (Onorato et al. 2009; Toffoli et al. 2010; Xiao et al. 2013; Fedele 2015; Annenkov \& Shrira 2009, 2018), yet as a result of very different underlying physics.

\section{Acknowledgement}

This work was funded by UK/China ORE funding (EPSRC/NERC/NSFC EP/R007632/1) and the De-Risk project funded by Innovation Fund Denmark. TSvdB acknowledges a Royal Academy of Engineering Research Fellowship.

\section{Declaration of interests}

The authors report no conflict of interest.

\section{REFERENCES}

AdCOCK, T. A. A. \& TAYLOR, P. H. 2009 Focusing of unidirectional wave groups on deep water: an approximate nonlinear Schrödinger equation-based model. Proc. R. Soc. A 465 (2110), 3083-3102.

Adcock, T. A. A., TAYlor, P. H. \& Draper, S. 2015 Nonlinear dynamics of wave-groups in random seas: unexpected walls of water in the open ocean. Proc. R. Soc. A Math. Phys. Eng. Sci. 471 (2184).

Annenkov, S. Y. \& Shrira, V. I. 2006 Role of non-resonant interactions in the evolution of nonlinear random water wave fields. J. Fluid Mech. 561, 181-207.

Annenkov, S. Y. \& Shrira, V. I. 2009 Evolution of kurtosis for wind waves. Geophys. Res. Lett. 36 (13).

Annenkov, S. Y. \& ShriRA, V. I. 2018 Spectral evolution of weakly nonlinear random waves: kinetic description versus direct numerical simulations. J. Fluid Mech. 844, 766795.

Baldock, T. E., Swan, C. \& Taylor, P. H. 1996 A laboratory study of nonlinear surface waves on water. Philos. Trans. R. Soc. A 354 (1707), 649-676.

Barratt, D., Bingham, H. B. \& Adcock, T. A. A. 2020 Nonlinear evolution of a steep, focusing wave group in deep water simulated with OceanWave3D. J. Offshore Mech. Arct. Eng. 142 (2).

Benjamin, T. B. \& Feir, J. E. 1967 The disintegration of wave trains on deep water Part 1. Theory. J. Fluid Mech. 27 (3), 417-430.

Bоссотті, P. 1983 Some new results on statistical properties of wind waves. Appl. Ocean Res. 5 (3), 134-140.

Bоссотті, P. 1989 On mechanics of irregular gravity waves. Atti Accademia Nazionale dei Lincei, Memorie viii (19), 111-170.

Bоссотті, P. 2000 Wave mechanics for ocean engineering. Elsevier.

Chabchoub, A. \& Grimshaw, R. 2016 The hydrodynamic nonlinear Schrödinger equation: Space and time. Fluids 1 (3), 23.

Dematteis, G., Grafke, T., Onorato, M. \& Vanden-Eijnden, E. 2019 Experimental evidence of hydrodynamic instantons: the universal route to rogue waves. Phys. Rev. $X 9$ (4), 041057.

Dudley, John M, Genty, Goëry, Mussot, Arnaud, Chabchoub, Amin \& Dias, Frédério 2019 Rogue waves and analogies in optics and oceanography. Nat. Rev. Phys. 1 (11), 675689.

Dyachenko, A. I. \& Zakharov, V. E. 2011 Compact equation for gravity waves on deep water. JETP Lett. 93 (12), 701. 
Dysthe, K. B. 1979 Note on a modification to the nonlinear Schrödinger equation for application to deep-water waves. Proc. R. Soc. A 369, 105-114.

Engsig-Karup, A. P., Bingham, H. B. \& Lindberg, O. 2009 An efficient flexible-order model for 3D nonlinear water waves. J. Comp. Phys. 228 (6), 2100-2118.

Fedele, F. 2014 On certain properties of the compact Zakharov equation. J. Fluid Mech. 748, $692-711$.

Fedele, F. 2015 On the kurtosis of deep-water gravity waves. J. Fluid Mech. 782, 25-36.

Fedele, F., Benetazzo, A., Gallego, G., Shih, P. C., Yezzi, A., Barbariol, F. \& Ardhuin, F. 2013 Spacetime measurements of oceanic sea states. Ocean Model. 70, 103115.

Fedele, F., Cherneva, Z., Tayfun, M. A. \& Guedes Soares, C. 2010 Nonlinear Schrödinger invariants and wave statistics. Phys. Fluids 22 (3), 036601.

Fedele, F. \& Dutykh, D. 2012 Special solutions to a compact equation for deep-water gravity waves. J. Fluid Mech. 712, 646-660.

GodA, Y. 2000 Random seas and design of maritime structures. World scientific.

Hasselmann, K. 1962 On the non-linear energy transfer in a gravity-wave spectrum. Part 1: General theory. J. Fluid Mech. 12, 481.

Janssen, P. A. E. M. 2003 Nonlinear four-wave interactions and freak waves. J. Phys. Ocean. 33 (4), 863-884.

Janssen, P. A. E. M. \& Bidlot, J. R. 2009 On the extension of the freak wave warning system and its verification. ECMWF Tech. Mem. $\mathbf{5 8 8}$.

Janssen, P. A. E. M. \& Janssen, A. J. E. M. 2019 Asymptotics for the long-time evolution of kurtosis of narrow-band ocean waves. J. Fluid Mech. 859, 790-818.

Kit, Eliezer \& Shemer, Lev 2002 Spatial versions of the Zakharov and Dysthe evolution equations for deep-water gravity waves. J. Fluid Mech. 450, 201-205.

Kokorina, A. \& Slunyaev, A. 2019 Lifetimes of rogue wave events in direct numerical simulations of deep-water irregular sea waves. Fluids 4 (2), 70.

Lindgren, G. 1970 Some properties of a normal process near a local maximum. Ann. Math. Statist 41 (6), 1870-1883.

Lo, E. \& MEI, C. C. 1985 A numerical study of water-wave modulation based on a higher-order nonlinear Schrödinger equation. J. Fluid Mech. 150, 395-416.

Longuet-Higgins, M. S. 1952 On the statistical distribution of the height of sea waves. J. Mar. Res. 11, 245-266.

Mori, N. \& Janssen, P. A. E. M. 2006 On kurtosis and occurrence probability of freak waves. J. Phys. Ocean. 36 (7), 1471-1483.

Mori, N., Onorato, M. \& Janssen, P. A. E. M. 2011 On the estimation of the kurtosis in directional sea states for freak wave forecasting. J. Phys. Oceanogr. 41, 1484-1497.

Mori, N., Onorato, M., Janssen, P. A. E. M., Osborne, A. R. \& Serio, M. 2007 On the extreme statistics of long-crested deep water waves: Theory and experiments. J. Geophys. Res. Oceans 112 (C9).

Onorato, M., Cavaleri, L., Fouques, S., Gramstad, O., Janssen, P. E. A. M., Monbaliu, J., Osborne, A. R., Pakozdi, C., Serio, M., Toffoli, A. \& Trulsen, K. 2009 Statistical properties of mechanically generated surface gravity waves: a laboratory experiment in a three-dimensional wave basin. J. Fluid Mech. 627, 235-257.

Onorato, M., Osborne, A. R., Serio, M. \& Cavaleri, L. 2005 Modulational instability and non-Gaussian statistics in experimental random water-wave trains. Phys. Fluids 17, 078101.

Onorato, M., Osborne, A. R., Serio, M., Cavaleri, L., Brandini, C. \& Stansberg, C. T. 2004 Observation of strongly non-Gaussian statistics for random sea surface gravity waves in wave flume experiments. Phys. Rev. E 70 (6), 067302.

Onorato, M., Osborne, A. R., Serio, M., Cavaleri, L., Brandini, C. \& Stansberg, C. T. 2006 Extreme waves, modulational instability and second order theory: wave flume experiments on irregular waves. Europ. J. Mech.-B/Fluids 25 (5), 586-601.

Onorato, Miguel, Proment, Davide, El, Gennady, Randoux, Stephane \& Suret, PIERre 2016 On the origin of heavy-tail statistics in equations of the Nonlinear Schrödinger type. Phys. Lett. A 380 (39), 3173 - 3177. 
Serio, M., Onorato, M., Osborne, A. R. \& Janssen, P. A. E. M. 2005 On the computation of the Benjamin-Feir Index. Nuovo Cim. della Soc. Ital. di Fis. C 28 (6), 893-903.

Shemer, L., Jiao, H., Kit, E. \& Agnon, A. 2001 Evolution of a nonlinear wave field along a tank: experiments and numerical simulations based on the spatial Zakharov equation. $J$. Fluid Mech. 427, 107-129.

Shemer, L. \& Sergeeva, A. 2009 An experimental study of spatial evolution of statistical parameters in a unidirectional narrow-banded random wavefield. J. Geophys. Res: Oceans $114(\mathrm{C} 1)$.

Shemer, Lev, Sergeeva, Anna \& Liberzon, Dan $2010 a$ Effect of the initial spectrum on the spatial evolution of statistics of unidirectional nonlinear random waves. J. Geophys. Res. Oceans 115 (C12).

Shemer, L., Sergeeva, A. \& Slunyaev, A. $2010 b$ Applicability of envelope model equations for simulation of narrow-spectrum unidirectional random wave field evolution: Experimental validation. Phys. Fluids 22 (1), 016601.

Slunyaev, AV \& Sergeeva, AV 2012 Stochastic simulation of unidirectional intense waves in deep water applied to rogue waves. JETP Lett. 94 (10), 779-786.

Tang, T., Tromans, P. S. \& Adcock, T. A. A. 2019 Field measurement of nonlinear changes to large gravity wave groups. J. Fluid Mech. 873, 1158-1178.

TAyfun, M. A. 1980 Narrow-band nonlinear sea waves. J. Geophys. Res: Oceans 85 (C3), $1548-1552$.

Tayfun, M. A. \& Fedele, F. 2007 Wave-height distributions and nonlinear effects. Ocean Eng. 34 (11-12), 1631-1649.

Toffoli, A., Gramstad, O., Trulsen, K., Monbaliu, J, Bitner-Gregersen, E. \& Onorato, M. 2010 Evolution of weakly nonlinear random directional waves: laboratory experiments and numerical simulations. J. Fluid Mech. 664, 313-336.

Tromans, P. S, Anaturk, A. R. \& Hagemeijer, P. 1991 A new model for the kinematics of large ocean waves-application as a design wave. 1st Int. Offshore and Polar Eng. Conf., Edinburgh, UK .

Trulsen, K., Kliakhandler, I., Dysthe, K. B. \& Velarde, M. G. 2000 On weakly nonlinear modulation of waves on deep water. Phys. Fluids 12 (10), 2432-2437.

XiaO, W., Liu, Y., Wu, G. \& Yue, D. K. P. 2013 Rogue wave occurrence and dynamics by direct simulations of nonlinear wave-field evolution. J. Fluid Mech. 720, 357-392.

Zavadsky, A., Benetazzo, A. \& Shemer, L. 2017 On the two-dimensional structure of short gravity waves in a wind wave tank. Phys. Fluids 29, 016601.

Zhang, H. D., Guedes Soares, C., Chalikov, D. \& Toffoli, A. 2016 Modeling the spatial evolutions of nonlinear unidirectional surface gravity waves with fully nonlinear numerical method. Ocean Eng. 125, 60-69.

Zhang, H. D., Guedes Soares, C. \& Onorato, M. 2014 Modelling of the spatial evolution of extreme laboratory wave heights with the nonlinear Schrödinger and Dysthe equations. Ocean Eng. 89, 1-9.

\section{Appendix A. Evolution of excess kurtosis}

In this appendix, we explicitly provide the equations we use to evaluate the analytical solutions shown in figure 2

\section{A.1. The NLS (Mori \& Janssen 2006)}

Mori \& Janssen (2006) present the evolution of excess kurtosis for the time evolution of spatially homogeneous unidirectional waves that are initially normally distributed in the form of a three-dimensional integral (their Eq. (14)), which is subsequently evaluated in the narrow-bandwidth and large-time limit for Gaussian spectra (their (28)). Without taking the large-time limit, but invoking the other two solutions, this integral can be evaluated in closed-form (Fedele et al. 2010) (see also Fedele (2015); Janssen \& Janssen 
$(2019))$ :

$$
\frac{C_{4, \mathrm{NLS}}^{d}(x)}{\mathrm{BFI}^{2}}=\frac{\pi}{3 \sqrt{3}}\left[1-\frac{6}{\pi} \operatorname{Im}\left(i \arcsin \frac{1+2 i \alpha}{2}\right)\right],
$$

where $C_{4, \mathrm{NLS}}^{d}$ is the dynamic excess kurtosis based on NLS, Im is the imaginary part, $\alpha=2 \nu^{2} x / \lambda_{0}$ and $\nu$ is the bandwidth of the frequency spectrum. To obtain (A 1 ), we have also converted from time to space (see Fedele et al. (2010)).

\section{A.2. The cDZ (Fedele 2014)}

Fedele (2014) also proposes a correction of $C_{4, \mathrm{NLS}}^{d}$ based on the compact Zakharov (cDZ) equation:

$$
C_{4, \mathrm{cDZ}}^{d}=C_{4, \mathrm{NLS}}^{d}\left(1-0.4 \nu_{k}^{2}\right) .
$$

Here, $\nu_{k} \approx 2 \nu$ is the spectral bandwidth of the wavenumber spectrum $S(k)$ and $\nu$ that of the frequency spectrum $S(f)$. Then,

$$
C_{4, \mathrm{CDZ}}^{d}=C_{4, \mathrm{NLS}}^{d}\left(1-1.6 \nu^{2}\right),
$$

for narrow-band waves ( $\operatorname{small} \nu$ ).

We do not use the full model derived in Fedele (2014), which is valid for waves evolving in time, but only the leading-order correction in bandwidth given here.

\section{Appendix B. Figure with BFI at steady state}

In figure 9 we show the same results as in figure 2 but with excess kurtosis normalised by at steady state $\mathrm{BFI}_{\mathrm{ss}}^{2}$. We thus obtain better agreement with theoretical results at large distances but worse agreement at small distances. Evidently, neither normalisation captures the occurrence of a maximum.

\section{Appendix C. Averaged shape of the wave with the largest crest-to-trough height}

The theory of quasi-determinism predicts the shape of the wave with the largest crestto-trough height as the scaled difference of two time-shifted autocovariance functions (Boccotti 1989, 2000).

Figure 10 (a) presents the average shape of the 5 largest crest-to-trough wave profiles at different locations. The general trend of the shape evolution is very similar to that of the largest crest events presented in figure 6 . We also observe a movement of the largest crest to the front of the wave group and a contraction of the wave group for extreme crest-to-trough events. Figure 10 (b) presents the predictions from the (linear) theory quasi-determinism given (Boccotti 1989, 2000). All the changes to the group shape in figure 10 (b) are due to changes in the wave spectrum. 

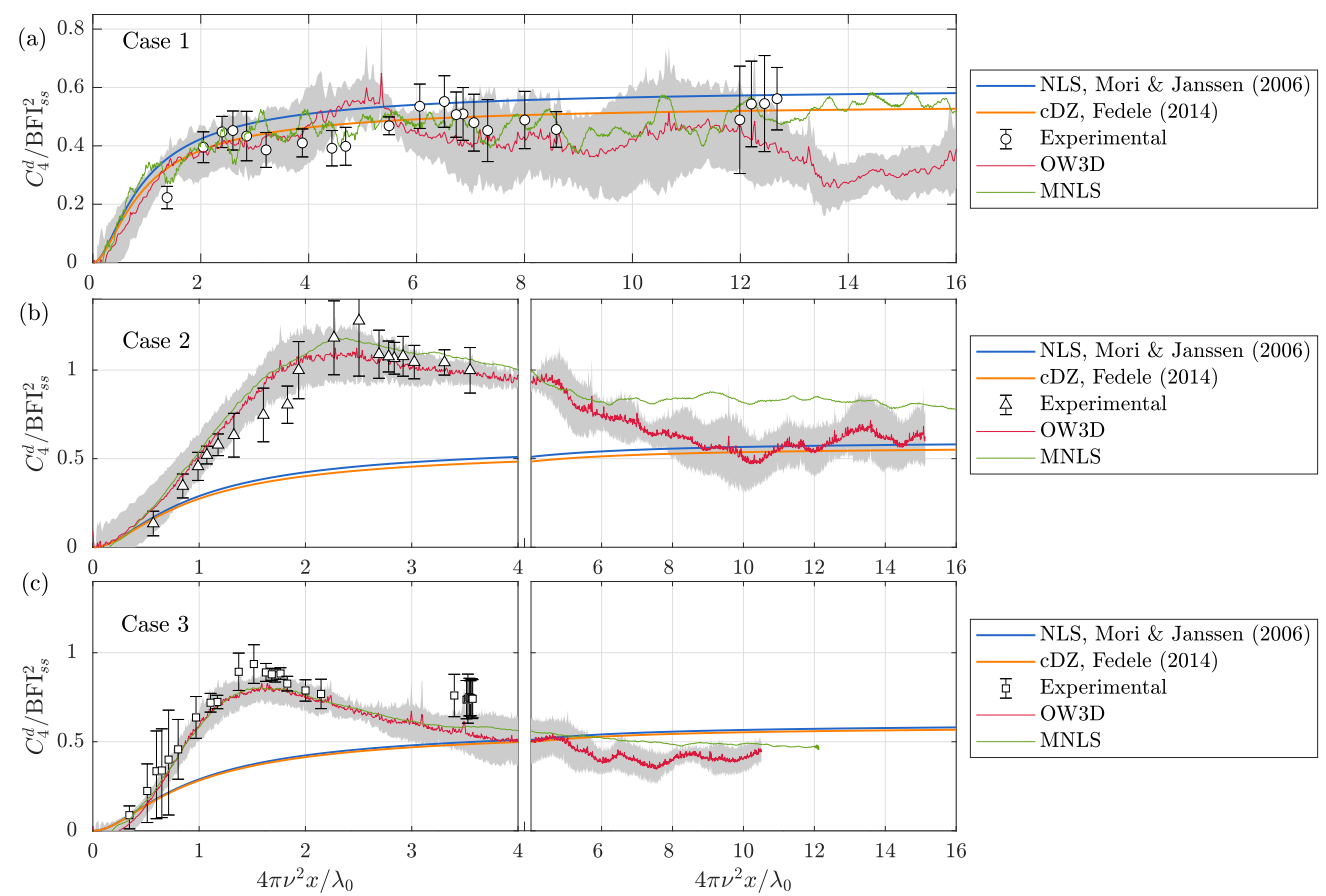

FiguRE 9. Evolution of normalised dynamic excess kurtosis at different distances from the wave generator: (a) case 1, (b) case 2 and (c) case 3. Shading represents the $95 \%$ confidence intervals for OceanWave3D simulation with 8 different random seeds. A total of 120 different random seeds are used in MNLS simulations. Consequently, for the MNLS, the error bars are negligible and have been omitted for clarity. The parameter $C_{4}^{d}$ is dynamic excess kurtosis, $\mathrm{BFI}_{s s}$ is the Benjamin-Feir Index at steady state, $\nu$ is the input bandwidth and $\lambda_{0}$ is the peak wave length. This figure is equivalent to figure 2 except for the normalisation by the steady-state (this figure) rather than the input BFI (figure 2). 


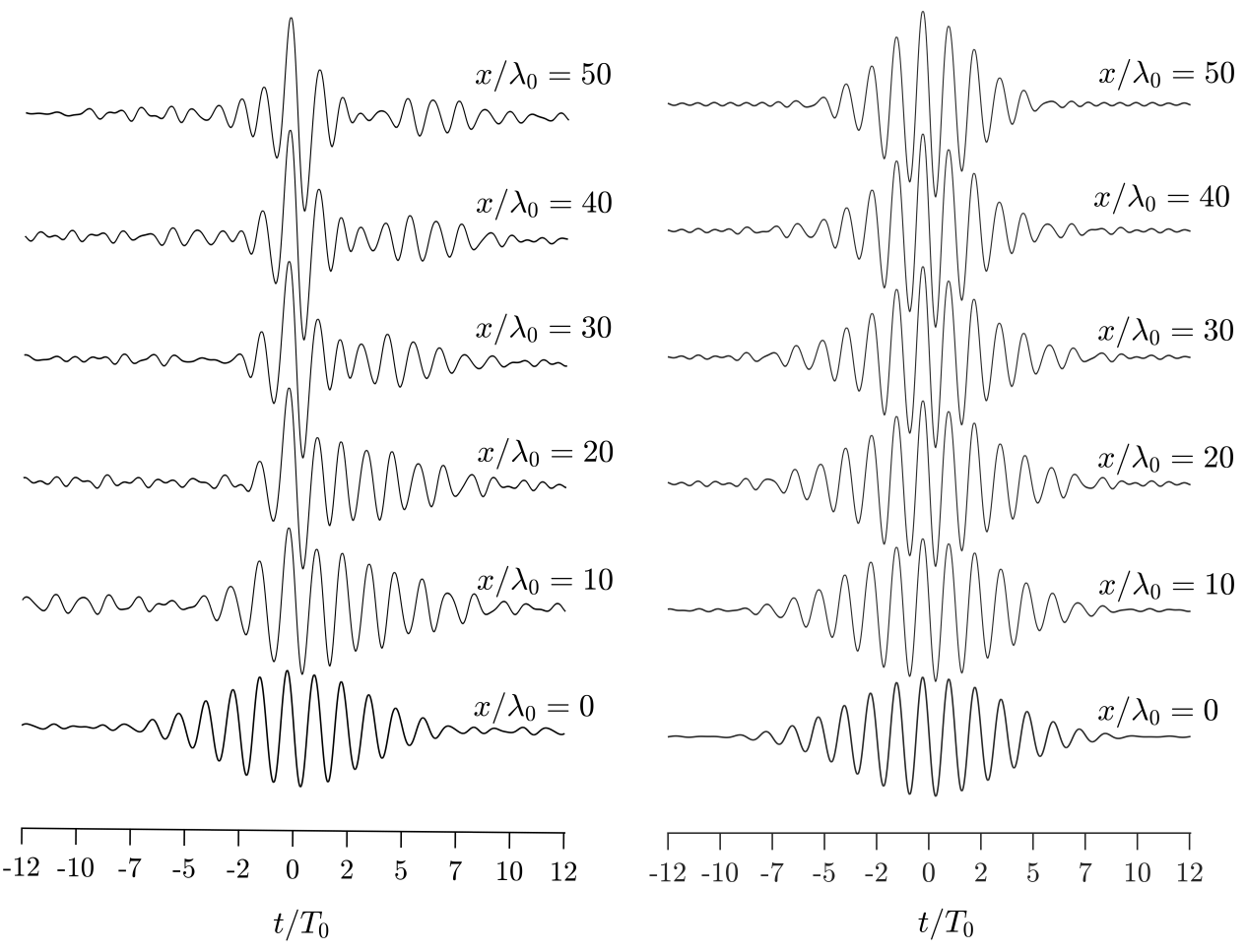

FiguRe 10. Average shape of the 5 largest crest-to-trough wave height profiles out of over 6400 waves profiles (left) and wave profiles predicted by the theory of quasi-determinism (right) at $x / \lambda_{0}=0,10,20,30,40,50$ for random waves with a Gaussian input spectrum with $\Delta f / f_{0}=0.054$ and $\epsilon=0.044$. 